\title{
NEMATOIDES GASTRINTESTINAIS DE EQUINOS COM ÊNFASE NO BIOCONTROLE POR Duddingtonia flagrans
}

(Gastrointestinal nematodes of equine with emphasis in biocontrole by Duddingtonia flagrans)

\author{
Andréia Buzatti ${ }^{1}$, Clóvis de Paula Santos², Douglas Luís Vieira' ${ }^{1}$, Marcelo Beltrão Molento ${ }^{1}$ \\ ${ }^{1}$ Programa de Pós-Graduação em Ciências Veterinárias. Setor de Ciências Agrárias. Universidade Federal do Paraná, ${ }^{2}$ Universidade \\ Estadual do Norte Fluminense, ${ }^{3}$ Programa de Residência em Área Profissional da Saúde em Medicina Veterinária. Universidade Federal do \\ Paraná \\ *Correspondência: deiabuzatti@gmail.com
}

RESUMO: O controle das infecções causadas por parasitos em equinos, em especial grandes e pequenos estrôngilos, desperta grande preocupação, pois estes podem causar graves prejuízos, ocasionando inclusive a morte dos animais acometidos. Somado a isto, o controle utilizado em criatórios baseia-se no uso intensivo de compostos químicos e sem associação com outras formas de controle, acelerando a seleção de populações parasitárias resistentes. Assim, existe a necessidade de métodos alternativos para o controle das infecções parasitárias em equinos. O controle biológico realizado com o fungo Duddingtonia flagrans tem apresentado resultados promissores. O fungo é capaz de resistir à passagem pelo trato gastrintestinal de equinos e desenvolver-se no material fecal, exercendo sua atividade predatória sobre estágios larvares de parasitos. Seu uso pode promover como consequência, a redução do número de larvas infectantes presentes na pastagem e uma menor taxa de reinfecção dos animais. Acreditamos que esta estratégia de controle parasitário para equinos possa ser associada a um programa sanitário integrado, reduzindo à dependência de anti-helmínticos químicos e a seleção para nematoides resistentes. Esta revisão tem o objetivo de abordar os principais helmintos em equinos e suas formas de controle, ressaltando o potencial de $D$. flagrans como alternativa no controle biológico dos parasitos.

Palavras-chave: controle biológico; helmintos; larvas infectantes; pastagem

ABSTRACT: The control of parasites infections in horses, especially large and small strongyles, are indispensable, because these agents can produce cause serious damages, causing even the death of the animals. The control strategy applied in the most of times is based exclusively on the use of chemical compounds, without association of other methods, accelerating the selection of resistant parasite populations. Biological control performed with the fungus Duddingtonia flagrans has demonstrated promising results as alternative method. The fungus is able to resist the passage through the gastrointestinal tract of horses. When eliminated with the feces and under favorable environmental conditions the fungus colonizes the dung and exerts predation on immature larval stages of parasitic nematodes. Their use can promote the reduction of the number of infective larvae on the pasture and consequently the rate of infection of the animals. Moreover, it can be inserted in an integrated program of parasite control, reducing the dependence to the anthelmintic compounds and the selection of resistant nematodes. This review aims to address the main helminthes in equines and their forms of control, highlighting the potential 
of $D$. flagrans as an alternative to the biological control of parasites.

Key Words: biological control; helminths; infective larvae; pasture

\section{INTRODUÇÃO}

As doenças parasitárias ocupam lugar de destaque entre os fatores que interferem no desenvolvimento pleno da produção animal, principalmente as causadas por endoparasitos, as quais apresentam elevada prevalência e ampla distribuição geográfica (Barret et al., 2004). Entre os endoparasitos de equinos, salvo o cestóide Anoplocephala perfoliata, são mais comuns os nematoides, Parascaris equorum, Oxyuris equi, Strongylus spp. (Proudman e Mattews, 2000) e os ciatostomíneos que são apresentam maior prevalência e importância (Gawor, 1995; Love et al. 1999). Os sistemas de criação, na maioria das vezes, favorecem uma elevada incidência dessas parasitoses já nas primeiras semanas de vida (Molento, 2005), ocasionando importantes condições patológicas tais como diarreia, cólicas e emagrecimento (Loon et al., 1995; Aluja et al. 2000) que podem levar a um retardo no desenvolvimento corporal e até a morte de animais seriamente infectados.

O controle de parasitos em equinos na maioria dos criatórios baseia-se exclusivamente no uso de compostos antiparasitários comerciais, por sua praticidade, alta eficiência, ótima relação custo-benefício e facilidade de aquisição. Porém, estes produtos são utilizados de forma supressiva e indiscriminada e sem a associação de estratégias de controle adequadas, ocasionando a seleção de populações parasitárias resistentes (Molento, 2005). Segundo Kaplan (2004), o aparecimento da resistência parasitária em equinos tem se tornado cada vez mais importante no decorrer dos anos, principalmente em relação ao controle inadequado, gerando um grande entrave econômico aos sistemas produtivos mundialmente.

Dessa forma, ressalta-se a importância da integração de outras formas de controle, principalmente buscando reduzir o número de larvas infectantes nas pastagens, promovendo assim a redução da reinfecção dos animais e consequentemente o uso de anti-helmínticos (Araújo et al., 2004; Braga et al., 2009). O controle biológico realizado com fungos nematófagos destaca-se como uma alternativa potencial ao controle das nematodioses em equinos, podendo ser facilmente aceito pelo mercado (Nielsen, 2012). Entre as espécies avaliadas com este objetivo, o Duddingtonia flagrans apresenta sólida comprovação em testes in vitro e in vivo em várias espécies animais (Larsen, 2000, 2006; Braga e Araújo 2014).

Esta revisão tem o objetivo de abordar os principais helmintos em equinos e suas formas de controle, ressaltando o potencial de $D$. flagrans como alternativa promissora para 0 controle biológico desses parasitas.

\section{BIOLOGIA E CICLO DE VIDA DE PEQUENOS $E$ GRANDES ESTRÔNGILOS}

A fauna parasitária de equinos é vasta, compreendendo várias famílias e gêneros, dentre os quais, os nematodas estrongilídeos se destacam como os mais prevalentes. Das 83 espécies descritas, a maioria (64) pertence à família Strongylidae e engloba os nematoides mais comuns e de importância econômica aos equinos (Lichtenfels et al. 2008). Esta família está dividida nas sub-famílias: 
Strongylinae que contem nematoides geralmente de tamanho médio a grande, possuindo capsula bucal globular ou em forma de funil e a Cyathostominae que têm geralmente tamanho pequeno a médio e com cápsula bucal cilíndrica (Lichtenfels et al. 2008). Strongylinae e Cyathostominae são vulgarmente designados de grandes e pequenos estrôngilos, respectivamente (Jacobs et al., 2016).

Grandes estrôngilos englobam os gêneros Strongylus, Oesophagodontus, Bidentostomum, Craterostomum e Triodontophorus, os quais parasitam o intestino grosso de equinos e asininos. As espécies do gênero Strongylus: $S$. vulgaris, $S$. equinus e $S$. edentatus apresentam como característica, processos migratórios complexos no organismo dos hospedeiros, enquanto os demais gêneros não são migratórios (Taylor et al., 2007).

Os pequenos estrôngilos, ou ciatostomíneos, correspondem a mais de 50 espécies, distribuídas em 14 gêneros: Cyathostomum, Coronocyclus, Cylicodontophorus, Cylicocyclos, Cylicostephanus, Skrjabinodentus, Tridentoinfudinbulum, Petrovinema, Poteriostomum, Gyalocephalus, Parapoteriostomum, Hsiungia, Cylindropharynx e Caballonema (Lichtenfels et al., 1998). Os ciatostomíneos possuem distribuição mundial e afetam animais de todas as idades (Corning, 2009), sendo presentes em 100 dos animais (Molento et al., 2008).

\section{PATOGENIA E SINAIS CLÍNICOS}

A infecção por grandes estrôngilos está associada a várias alterações clínicas, devido ao processo migratório das larvas e adultos na parede intestinal e vasos sanguíneos (Bellaw et al., 2016). Neste grupo, $S$. vulgaris é considerada a espécie mais patogênica, principalmente na sua forma imatura, em decorrência das lesões que causa durante o processo de migração pelo sistema arterial mesentérico, podendo ser responsável por quadros de cólica intestinal severa (Bellaw et al., 2016; Nielsen et al., 2014; Reinemeyer et al., 2014). A migração de fases préadultas de $S$. edentatus está associada a alterações macroscópicas no fígado e presença de nódulos em tecidos subperitoneais, podendo ocorrer diarreia, hipertermia, edema, anorexia, depressão e perda de peso. As larvas migratórias de $S$. equinus apresentam comportamento invasivo, porém sua patogenia ainda não é totalmente conhecida, apresentando sinais clínicos semelhantes aos descritos para $S$. edentatus (Taylor et al., 2007).

A patogenia da infecção por parasitas adultos está associada aos danos causados à mucosa do intestino grosso, pois os adultos não desenvolvem atividade migratória sistêmica ativa. Os danos são promovidos pelos hábitos alimentares dos parasitos e também pela emergência de adultos jovens no intestino, provocando lesões na mucosa e vasos sanguíneos com subsequente perda de sangue, líquidos teciduais e nutrientes. Esses fatores são os principais responsáveis por sinais de debilidade e anemia (Taylor et al., 2007).

Nas infecções por pequenos estrôngilos, a saída simultânea de grande número de larvas, especificamente as de quarto estádio (L4), da mucosa e submucosa intestinal pode ocasionar a síndrome de migração larval, também conhecida como "ciatostomíase larval". As larvas e adultos na luz do órgão causam uma enteropatia inflamatória significativa no ceco e cólon, resultando em enterite catarral e hemorrágica, diarreia, cólica, perda de peso, podendo muitas vezes levar o animal a óbito (Cobb e Boeckh, 2009). Enquanto um grande número de parasitos adultos pode causar sinais 
como letargia, perda de peso, atraso no desenvolvimento, debilidade e diarreia (Corning, 2009), potros e animais jovens costumam apresentar mais sinais clínicos. Em infecções leves, os animais podem não apresentar sinais clínicos, convivendo com uma baixa carga parasitária. A colite granulomatosa também é uma patologia que tem sido associada às larvas de ciatostomíneos (Love et al., 1999).

\section{CICLO BIOLÓGICO EPIDEMIOLOGIA}

As espécies da família Strongylidae apresentam ciclo de vida direto, com uma fase exógena e estádios larvares no ambiente. As condições ambientais, para o desenvolvimento e sobrevivência das formas de vida livre, são idênticas para as duas subfamílias (Reinemeyer, 2009). O ovo é eliminado nas fezes do hospedeiro e se desenvolve no ambiente, passando por dois estádios larvais intermediários (L1 e L2) até atingir a fase infectante, ou L3. Na fase endógena ocorre o estádio $L 4$, antes da maturação para adulto, que envolve algumas migrações no interior do hospedeiro (Madeira de Carvalho, 2003). O número de ovos e larvas viáveis no meio-ambiente é condicionado por diversos fatores, sendo a temperatura ambiental e a umidade relativa do ar os mais importantes. Além da concentração de oxigênio, luz solar, tipo de cobertura vegetal, predadores, organismos coprófagos e os fatores abióticos que podem estar presentes nas fezes dos equídeos (Madeira De Carvalho, 2003).

No Brasil, as pastagens apresentam condições favoráveis à sobrevivência de larvas de nematoides gastrintestinais durante todas as estações do ano, sendo que o bolo fecal, quando presente no pasto exerce a função de reservatório, protegendo as L3 da dissecação, fazendo com que algumas perdurem por vários meses, ou até mesmo por mais de um ano. Estas características indicam claramente a presença constante de larvas e o risco de infecção dos animais. Quando em condições ambientais favoráveis, as larvas abandonam a massa fecal migrando horizontal e verticalmente para a fração da vegetação que será ingerida pelo hospedeiro, dando continuidade ao ciclo (Kuzmina et al., 2006). Dados nacionais indicam que o conhecimento da epidemiologia desses parasitos é de extrema importância para a implementação de formas adequadas de controle (Souza et al., 2012; Molento et al., 2016).

\section{PRÁTICAS DE CONTROLE PARASITÁRIO: MEDICAMENTOS ANTIPARASITÁRIOS}

A forma de controle adotado na maioria das criações de equinos se baseia exclusivamente no uso de compostos antiparasitários, principalmente por sua praticidade e eficiência, ótima relação custo-benefício e pela facilidade de aquisição (Molento, 2005). Dentre os produtos disponíveis, existem três grupos químicos distintos que são os mais utilizados: os benzimidazóis, as pirimidinas, e as lactonas macrocíclicas. Sabe-se que a alternância entre esses grupos químicos é frequente, ocorrendo muitas vezes ao ano (Traversa, 2008; Canever et al., 2013).

Os antiparasitários são usados de forma supressiva, curativa ou estratégica nas várias formas de criação equina. $O$ tratamento supressivo é administrado em intervalos curtos, principalmente em potros de até 18 meses, já o estratégico está associado com a época do ano e com o aumento do número de parasitas no animal, com o uso da OPG e obedecendo a epidemiologia dos parasitos. $\mathrm{O}$ tratamento supressivo foi preconizado na década de 60 por (Herd, 1990; 
Nielsen, 2009) e envolve o tratamento de todos os animais em intervalos curtos (seis a oito semanas). Este método se tornou o mais comumente utilizado, sendo ainda hoje seguido por proprietários. Outra abordagem é o tratamento estratégico, que tem o objetivo de tratar os animais em épocas do ano pré-determinadas, de acordo com o conhecimento prévio da flutuação de larvas nas pastagens (Courtney e Asquith, 1985; Herd, 1985). No tratamento curativo o tratamento é aplicado quando o animal apresenta alta contagem de ovos nas fezes ou sinais clínicos (Sangster et al., 2002). Uma das formas de tratamento curativo é o uso seletivo de anti-helmínticos. Sabendo-se que muitos equinos abrigam baixas cargas parasitárias, enquanto outros carregam cargas mais moderados e que alguns indivíduos são fortemente infectados com parasitos, apenas aqueles que necessitarem de tratamento serão vermifugados (Nielsen, 2012). Neste caso, o critério para se tratar foi baseado na contagem do número de ovos por grama de fezes (OPG), ou seja, animais com OPG acima de 200.

Estas modalidades não têm sido capazes de deter o avanço da resistência anti-helmíntica e acabam contribuindo para pressão de seleção a nematoides resistentes. Kaplan (2002) sugeriu que grande parte dos criadores de cavalos possui elevada preocupação em relação ao impacto das parasitoses. Esta concepção geralmente resulta na busca por um controle "total" das parasitoses. Para obter tal objetivo, costuma-se adotar o tratamento supressivo, esperando que a contagem de OPG próximas de zero signifique manter a carga parasitária na mesma concentração. Porém, deve-se ter em mente que a utilização indevida desses produtos resulta em eficácias abaixo da expectativa, aumentando os custos de produção (Cezar et al., 2008) e o processo de seleção de parasitas resistentes e consequentemente diminuindo a vida útil dos antiparasitários (Geurden et al., 2014). Canever et al. (2013) relataram que $100 \%$ das criações de cavalos Puro Sangue Inglês no Paraná, São Paulo e Santa Catarina, avaliados apresentaram baixa eficácia do fenbendazole.

Inicialmente devemos reconhecer que o controle das doenças parasitárias em equinos passa por um momento de reflexão, isto considerando somente o início das descobertas de resistência dos parasitas no mundo (Peregrine et al., 2014). Outro ponto para reflexão é abdicar do conceito de se ter um animal com "cura total", prometendo $100 \%$ de eficácia dos produtos. Molento (2009) sugeriu que o controle parasitário deve ter como base novas estratégias de gestão, buscando reduzir o uso de antihelmínticos, a seleção de parasitas resistentes e também a liberação de produtos químicos no ambiente. Concomitantemente, deve-se garantir a exposição dos animais a certos níveis dos agentes, permitindo dessa forma que animais jovens possam desenvolver uma adequada imunidade. Este raciocínio vem contribuir para a identificação de animais mais tolerantes contra as parasitoses, o que poderá ser considerado em programas de reprodução e melhoramento.

Muito embora as causas parasitárias sejam consideradas causadoras de redução no desempenho de equinos, existem poucas evidencias quando se trata de animais de uma mesma raça, provenientes da mesma propriedade e que recebem os mesmos cuidados sanitários. O obvio é reconhecer que animais que exercem uma atividade de trabalho pesado, ou que nascem em situação de baixa renda e não recebam cuidados básicos, podem ser acometidos com alta carga parasitária e apresentar crescimento reduzido. Entretanto, animais de 
propriedades de alto padrão, têm apresentado índices de mortalidade relativamente elevados quando há existência de surtos parasitários, mesmo recebendo tratamentos com medicação de amplo-espectro (Peregrine et al., 2014).

Além disso, recomenda-se a integração de outras formas de controle, buscando reduzir o número de L3 nas pastagens, promovendo assim a redução da reinfecção dos animais e consequentemente 0 uso de antihelmínticos (Araújo et al., 2004; Braga et al., 2009). Nesse sentido, o controle biológico realizado com fungos nematófagos destaca-se como uma alternativa promissora, podendo ser utilizado em combinação com o controle parasitário convencional (Braga et al., 2010).

Tratando-se da redução do uso de anti-helmínticos a terapia seletiva dos animais enquadra-se como uma estratégia, principalmente em relação à resistência. Estudos têm sido desenvolvidos com o objetivo de avaliar o ponto de corte ideal de OPG e a aplicabilidade de tratamento antiparasitário para cada espécie e categoria animal. Além da contagem de OPG outros fatores/técnicas como avaliação clínica do animal e coprocultura, devem ser associados para adequada realização da terapia seletiva (Nielsen et al., 2006). Kaplan e Nielsen (2010) afirmam que a eficácia da abordagem de terapia seletiva para o controle de parasitas de equinos ainda é limitada e a maioria dos estudos avalia apenas uma abordagem em curto prazo. No entanto, muita evidência existe comprovando a inadequação nas abordagens de rotação tradicional. $\mathrm{O}$ valor corte de OPG para tratamento médio atualmente é de 200 OPG (Nielsen et al., 2006; Lloyd, 2009; Kaplan e Nielsen, 2010) esse fato parece resultar da publicação de Uhlinger (1993), onde diferentes laboratórios foram questionados sobre seus pontos de corte recomendados. No Brasil, este tratamento tem sido utilizado desde 2005 e apresenta vantagens na relação custo-benefício, na melhoria da avaliação clínica dos animais e na manutenção da eficácia dos medicamentos. O chamado ponto-decorte foi elevado para 400 OPG para potros e de 600 para éguas (Abrahão, 2016).

Assim, existe claramente uma diferença na atitude dos profissionais envolvidos com a saúde em cavalos que por si só, reflete na qualidade e longevidade do controle parasitário.

\section{PRÁTICAS DE CONTROLE PARASITÁRIO: FUNGOS NEMATÓFAGOS}

Os fungos nematófagos estão presentes na natureza como antagonistas naturais de nematoides de vida livre, sendo conhecidos agentes destruidores de nematoides (Barron, 1977) e considerados uma estratégia promissora para o controle biológico de nematoides (Braga e Araújo, 2014). Estes organismos foram primeiramente descritos há mais de um século, catalogados em mais de 150 espécies, as quais se encontram divididas em três grupos principais: oportunistas, endoparasitos e predadores (Barron, 1977; Gronvold et al.,1993).

Os fungos oportunistas parasitam ovos, iniciando seu mecanismo de ação pela colonização da superfície dos ovos e posterior penetração das hifas por poros presentes na membrana dos mesmos. O processo de penetração ocorre por um conjunto de ações mecânicas e enzimáticas (Lysek e Sterba, 1991) e esta ação ocasiona aumento da permeabilidade da membrana da casca, facilitando a passagem de toxinas promovendo 0 desequilíbrio osmótico, que prejudica diretamente 0 desenvolvimento dos estágios pré-parasitários (Stirling e 
Oeste, 1991). O fungo pode ainda colonizar o conteúdo interno do ovo e afetar a larva em desenvolvimento (Mota et al., 2003).

Os fungos endoparasitos atuam em estádios larvares e também em nematoides adultos. $O$ processo de infecção inicia-se pela ingestão de esporos capazes de se desenvolver e colonizar o conteúdo interno dos nematoides, podendo promover a morte dos mesmos (Barron, 1977). Já os predadores pertencem a um grupo heterogêneo de microfungos, caracterizado por sua habilidade em capturar e destruir nematoides por meio de hifas modificadas, assim como competir pelas fontes de nutrientes ou suplementos necessários para 0 desenvolvimento fúngico no meio ambiente (Waller e Faedo, 1996).

$$
\text { Os fungos predadores }
$$

caracterizam-se pela produção de um sistema de hifas semelhante a armadilhas, onde capturam formas larvares de nematoides parasitas em fase exógena de desenvolvimento, ou nematoides de vida livre (Figura 1).

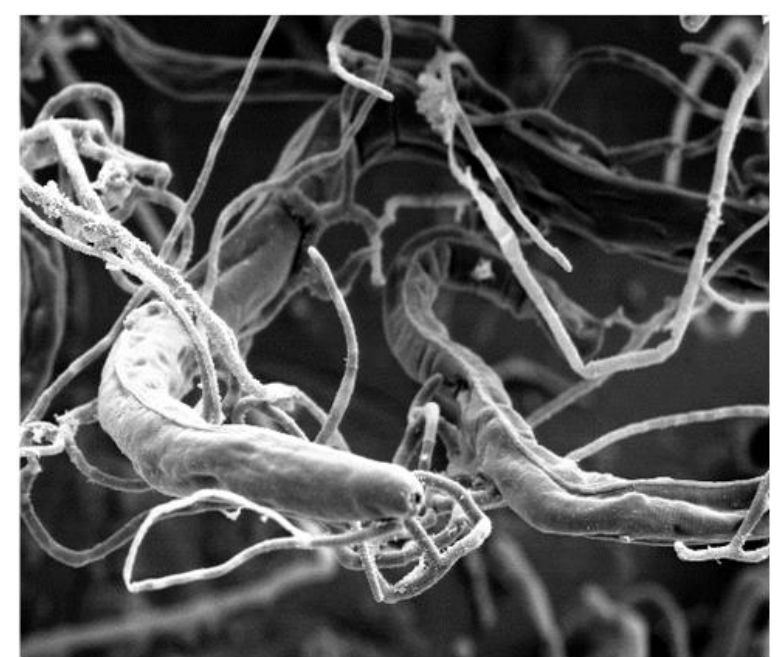

Figura 1 - Sistema de hifas (estrelas) de fungos predadores contendo ao longo de algumas destas formações que funcionam como armadilhas. No detalhe, Panagrellus spp. (setas) aprisionado por redes tridimensionais de D. flagrans (ponta de seta). Barra: $50 \mu$

Existem vários tipos destas armadilhas que agem por ação mecânica ou adesiva sendo divididas em hifas adesivas não modificadas ou não diferenciadas, ramificações hifais anastomosadas formando redes adesivas tridimensionais, ramificações adesivas que algumas vezes formam redes simples e na maioria das vezes bidimensionais, nódulos adesivos, anéis constritores e anéis não constritores (Barron, 1977). O estímulo para produção dessas armadilhas pode acontecer em função da motilidade (Jansson e Nordbring-Hertz, 1980), concentração (Gronvold, 1989) e substâncias oriundas de nematoides (Wotton e Pramer, 1966; Nordbrin-Hertz, 1973; Burney e Estey, 1985) condições adversas, tais como: escassez de nutrientes e de água (Balan e Gerber, 1972) ou até mesmo espontaneamente no caso de alguns isolados (Hayes e Blackburn, 1966). O aparecimento das estruturas predadoras ocorre de $30 \mathrm{~min}$ até $4 \mathrm{~h}$ após a interação fungonematoide. No caso de L3 dos nematoides parasitos, a cutícula externa pode retardar o processo de infecção, porém não confere proteção à ação predatória dos fungos (Cruz et al., 2011).

Segundo Dias et al. (2007), os fungos classificados como predadores destacam-se dentre as alternativas mais promissoras para o controle biológico das doenças parasitárias dos animais domésticos. Dentre outros fatores, estão naturalmente em abundância no solo e podem utilizar vários substratos orgânicos e micro habitats que promovem oportunidades para a interação com nematoides (Gronvold et al.,1996). Estes fungos são cosmopolitas e além do solo, podem ser encontrados em pastagens e detritos orgânicos em decomposição, juntamente com estágios imaturos de nematoides parasitas (Faedo et al., 1998). Mota et al. (2003) esclarecem que esses agentes não apresentam efeito sobre as fases parasitárias dos nematoides, concentrando suas ações 
sobre os hospedeiros intermediários, paratênicos, vetores e estádios larvais de vida livre, diminuindo a fonte de infecção para seus hospedeiros finais. Desta forma, para animais criados em sistemas extensivos, o uso do fungo como terapia alternativa, pode propiciar a redução de L3 presentes na pastagem e consequentemente a taxa de reinfecção dos animais.

Um requisito essencial para que um isolado de fungo seja possivelmente explorado no controle biológico de parasitos, é que ele possua a habilidade de suportar a passagem pelo trato gastrintestinal dos animais após administração oral. Ou seja, deve tolerar condições de estresse e, uma vez presente nas fezes deve ser capaz de germinar, colonizar o bolo fecal e capturar os estágios larvares eclodidos dos ovos, antes que estes migrem para fora do bolo fecal (Braga et al., 2010). Além disso, deve possuir especificidade de ação, alta capacidade reprodutiva e suportar as condições ambientais e climáticas da região geográfica onde o controle será realizado (Braga et al., 2009).

\section{PRÁTICAS DE CONTROLE PARASITÁRIO: USO DE Duddingtonia flagrans}

Duddingtonia flagrans é um fungo predador de nematoides e se destaca como um dos agentes mais estudados para uso no controle biológico das parasitoses dos animais domésticos (Braga et al., 2009), muito dessa importância se deve a sua elevada capacidade de sobrevivência ao trânsito gastrintestinal dos animais que é atribuída sobretudo, a produção intensa de clamidósporos que são estruturas de resistência superiores aos conídios (Faedo et al., 1997; Cruz et al., 2008).

A atividade predatória de $D$. flagrans inicia-se com a interação larva/nematoide de vida livre e fungo, promovendo contato entre as armadilhas e a cutícula dos nematoides, seguido do seu aprisionamento. Após isto, ocorre rompimento da cutícula, a entrada e o crescimento das hifas no interior do corpo da larva e toda esta ação ocasiona sua imobilização e digestão do seu conteúdo interno, causando a morte da larva (Campos et al., 2008; Cruz et al., 2011). O processo é complexo, ocorrendo por meio de uma combinação de eventos físicos e enzimáticos (Cruz et al., 2011). A ação enzimática é fundamental para a perfuração e degradação da cutícula de larvas infectantes de nematoides gastrintestinais. $\mathrm{Em} \quad D$. flagrans hidrolases do tipo fosfatase ácida (agem sobre ligações ésteres) e uroquinase um tipo de serino protease (agem sobre ligações peptídicas) demonstraram-se ativas durante a interação fungo nematoide (Cruz et al. 2009; Cruz et al., 2015).

Duddingtonia flagrans produz clamidósporos, que são estruturas reprodutivas com parede espessa e altamente resistentes, diferenciados a partir das hifas, os quais podem dar origem a hifas, conidióforos e conídios (Barron, 1977). Se ingeridos, os clamidósporos percorrem 0 trato gastrintestinal dos animais e permanecem viáveis quando nas fezes, colonizando-as logo após a sua deposição no solo (Faedo et al., 1997). No bolo fecal, o desenvolvimento do fungo é estimulado pelo contato com um número crescente de larvas infectantes (Paz-Silva et al., 2011) e também por uma variedade de nematoides de vida livre. Com a colonização, os clamidósporos podem atingir a pastagem, sendo frequente sua ingestão juntamente com o pasto (Mota et al. 2003).

Em relação a possíveis efeitos adversos da administração de $D$. flagrans aos animais, Madeira de Carvalho (2007), concluíram, por meio de avaliação de parâmetros 
hematológicos e bioquímicos que o fungo não causa efeitos adversos quando administrado via oral à equinos. Além disso, poderia haver preocupação sobre outras consequências, como por exemplo, ação sobre outros organismos que trazem benefícios para o solo e para o meio ambiente. Porém, em estudo desenvolvido por Knox et al. (2002) foi comprovado que $D$. flagrans não promoveu impacto ambiental negativo, quando usado em um sistema de produção a pasto. Os autores observaram que o número de nematoides de vida livre e de microartrópodos não foram reduzidos $(P>0,05)$ pelo fungo, como também não foi verificado efeito $(P>0,05)$ sobre a presença de outros fungos predadores.

\section{PESQUISAS Duddingtonia flagrans: AÇÃO EM EQUINOS}

Desde o início da década de 90 até os dias atuais, os estudos desenvolvidos com uso de $D$. flagrans no controle das endoparasitoses em animais domésticos têm aumentado, incluindo estudos com equinos. Nas mais variadas regiões do mundo, pesquisadores relataram a eficácia de $D$. flagrans no controle de estágios imaturos de parasitos de bovinos (Larsen et al., 1995; Silva et al., 2013), caprinos e ovinos (Larsen et al., 1998; Waghorn et al., 2003; Ojeda-Robertos et al., 2008) e também de equinos (Bird e Herd, 1995; Larsen et al., 1996). A atividade predatória sobre nematoides de equinos já foi estudada em estes in vitro e a campo, com excelentes resultados, conforme dados apresentados na Tabela 1.

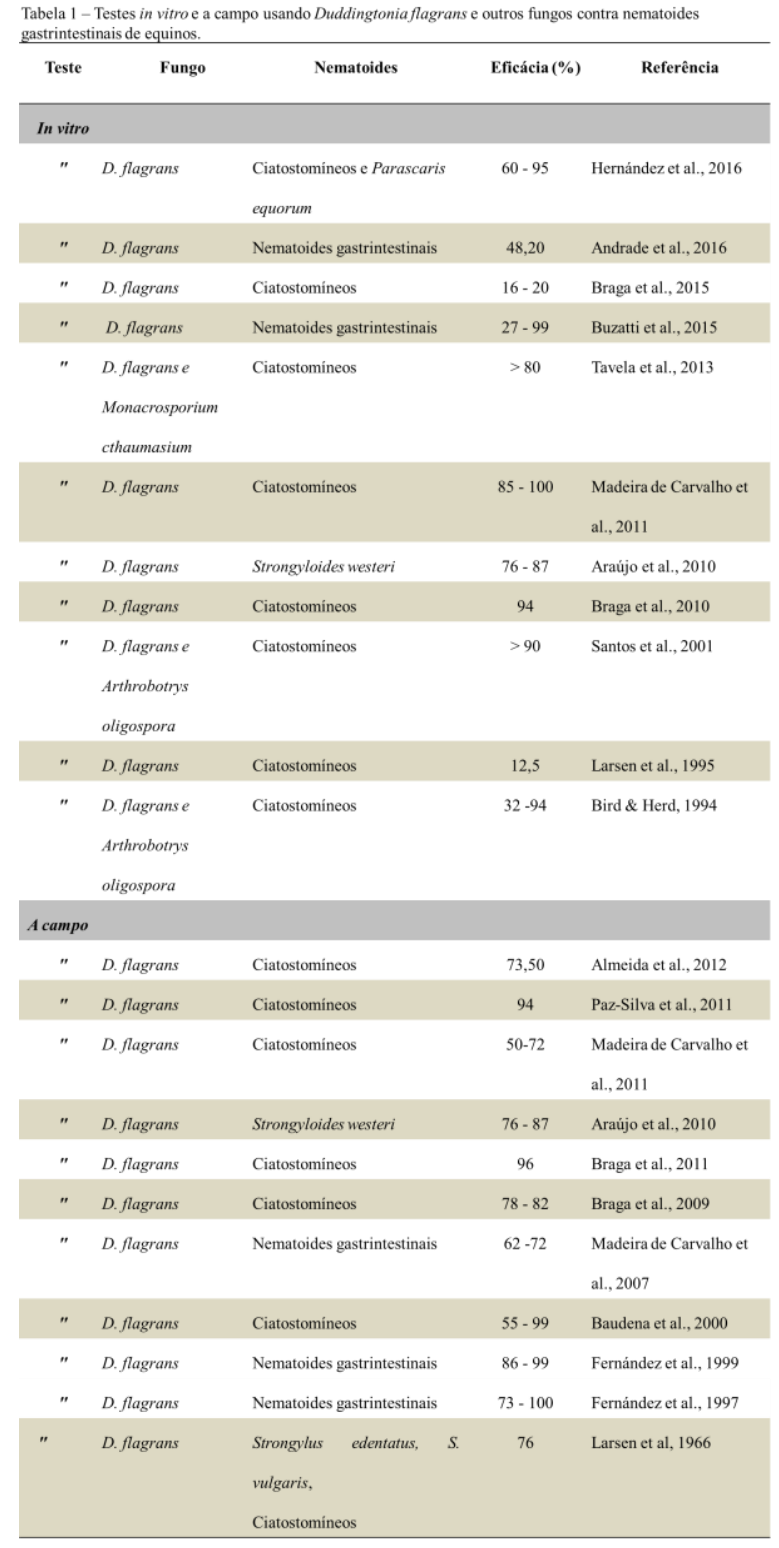

Um desafio ao uso do fungo como alternativa de controle biológico é a sua capacidade de resistência, desenvolvimento e atividade predatória em condições ambientais naturais, as quais, em muitos momentos podem ser adversas. Nesse sentido, estudos foram desenvolvidos a fim de se avaliar a eficácia do fungo na redução de L3 na pastagem após a passagem pelo trato gastrintestinal dos animais e sua eliminação juntamente com o bolo fecal. Fernández et al. (1997; 1999), em 4 meses de avaliações, observaram eficácias de 65 a 95\% e de 85,8 a 99,4\%, respectivamente. Em experimento realizado durante 12 
meses, Baudena et al. (2000) constataram redução de 66 a $99 \%$. Madeira de Carvalho et al. (2007) obtiveram 50 a $70 \%$ de eficácia em duas etapas de 6 meses. Em estudos com 6 meses de duração, Braga et al. (2009) e Almeida et al. (2012) observaram $78,5 \%$ e $73,5 \%$ de eficácia na redução de larvas infectantes na pastagem, respectivamente, o que se refletiu na diminuição do número de parasitos presentes no trato gastrintestinal dos animais que foram mantidos nos piquetes de experimentação.

Os excelentes resultados já observados e as pesquisas com a epidemiologia, biologia e modo de ação de fungos nematófagos demonstraram a viabilidade do emprego desses agentes para 0 controle de nematoides gastrintestinais de equinos. Entretanto, deve-se considerar a importância de avaliar os isolados com características predatórias promissoras em condições climáticas variadas (Mota et al., 2003). Duddingtonia flagrans, se desenvolveu melhor entre 20 e $25^{\circ} \mathrm{C}$ e índice pluviométrico entre 15 e 60 $\mathrm{mm}^{3} / \mathrm{semana}$ (Gronvold et al.,1996). Entretanto, essas condições podem variar conforme o isolado. Santos et al., (2001) observaram que no intervalo de 25 a $30^{\circ} \mathrm{C}, D$. flagrans promoveu uma redução de $L 3$ de ciatostomíneos superior a $90 \%$. Sendo $25^{\circ} \mathrm{C}$ a temperatura que proporcionou o maior índice de desenvolvimento de ovo até L3.

Madeira de Carvalho et al. (2007), afirmaram que um fator imposto a utilização de $D$. flagrans no controle biológico de parasitas é a necessidade de haver uma razoável presença do fungo nas fezes dos hospedeiros para que ocorra a redução das formas larvares no bolo fecal e consequentemente na pastagem. Nesse sentido, quando um animal ingere clamidósporos, ocorre a emissão dos mesmos juntamente com o conteúdo fecal. As fezes frescas de equinos, em condições climáticas ideais, são rapidamente colonizadas pelo fungo, sendo também frequente a sua ingestão durante o pastoreio, com subsequente excreção nas fezes (Larsen, 2000).

\section{CONCLUSÃO}

Diante da alta prevalência das endoparasitoses em equinos e levandose em consideração as falhas do controle químico, torna-se evidente a necessidade de métodos alternativos de controle para esta espécie animal. Como relatado, pesquisas com $D$. flagrans têm demonstrado resultados promissores no controle de nematoides gastrintestinais de equinos, sendo que 0 emprego desse fungo como estratégia de controle parasitário, pode diminuir a contaminação da pastagem e em médio e longo prazo, diminuir a ingestão de larvas infectantes e reduzir o desafio parasitário. Além disso, a presença do fungo poderá reduzir a dependência aos anti-helmínticos e a pressão de seleção para resistência parasitária.

\section{REFERENCIAS}

ALMEIDA, G.L.; SANTURIO, J.M.; JARDIM FILHO, J.O. et al. Predatory activity of the fungus Duddingtonia flagrans in equine strongyle infective larvae on natural pasture in the Southern Region of Brazil. Parasitology Research, v.110, n.2, p.657-662, 2012.

ALUJA, A.S., LÓPEZ, A., CHAVIRA, H. et al. Condiciones patológicas más frecuentes en los équidos de trabajo en el campo mexicano. Veterinária México, v.31, n.2, p.165, 2000.

ANDRADE M.C.; HIURA E.; DA FONSECA L.A. et al. Utilization of the Fungus Duddingtonia flagrans in Control of Nematode Larvae Development in Equine Stool. International Journal of Current Microbiology and Applied Sciences, v.5, n.3, p.829-35, 2016. 
ARAÚJO, J.V.D.; GUIMARÃES, M.P.; CAMPOS, A.K. et al. Control of bovine gastrointestinal nematode parasites using pellets of the nematode-trapping fungus Monacrosporium thaumasium. Ciência Rural, v.34, n.2, p.457-463, 2004.

ARAÚJO, J.M.; ARAÚJO, J.V.; BRAGA, F.R. et al. In vitro predatory activity of nematophagous fungi and after passing through gastrointestinal tract of equine on infective larvae of Strongyloides westeri. Parasitology Research, v.107, n.1, p.103-108, 2010.

BALAN, J.; GERBER, N. Attraction and killing of the nematode Panagrellus redivivus by the predacious fungus Arthrobotrys dactiloides. Nematology, v.18, p.163-173, 1972.

BARRET, E.J.; FARLAM, J.; PROUDMAN, C.J. Field trial of the efficacy of a combination of ivermectin and praziquantel in horses infected with roundworms and tapeworms. Veterinary Record, n.184, p.323-325, 2004.

BARRON, G.L. The Nematode destroying Fungi. In_ Topics in Mycobiology. Guelph, Canada. Ed. Canadian Biological Publications Ltda. 1977, $140 \mathrm{p}$.

BAUDENA, M.A.; CHAPMAN, M.R.; LARSEN, $M$. et al. Efficacy of the nematophagous fungus Duddingtonia flagrans in reducing equine cyathostome larvae on pasture in south Louisiana. Veterinary Parasitology, v.89, n.3, p.219-230, 2000.

BELLAW, J.L.; PAGAN, J.; CADELL, S. et al. Objective evaluation of two deworming regimens in young thoroughbreds using parasitological and performance parameters. Veterinary Parasitology, v.221, p.69-75, 2016.

BIRD. J.; HERD, R.P. In vitro assessment of two species of nematophagous fungi (Arthrobotrys oligospora and Arthrobotrys flagrans) to control the development of infective cyathostome larvae from naturally infected horses.

Veterinary

Parasitology, v.56, p.181-187, 1995.

BURNEY, K.; ESTEY, R. A note on the non diffusibility of a substance produced by nematodes that induced Arthrobotrysoligospora to form nematode traps. Phytoprotection, v.66, n.2, p.163-164, 1985.

BRAGA, F.R.; ARAÚJO, J.V.; SILVA, A.R. et al. Biological control of horse cyathostomin

Cyathostominae) using the nematophagous fungus Duddingtonia flagrans in tropical southeastern Brazil. Veterinary Parasitology, v.163, n.4, p.335-340, 2009.

BRAGA, F.R.; ARAÚJO, J.V.; SILVA, A.R. et al. Predatory activity of the nematophagous fungus Duddingtonia flagrans on horse cyathostomin infective larvae. Tropical animal health and production, v.42, n.6, p.1161-1165, 2010.

BRAGA, F.R.; ARAÚJO, J.V. Nematophagous fungi for biological control of gastrointestinal nematodes in domestic animals. Applied Microbiology and Biotechnology, v.98, n.1, p.71-82, 2014.

BRAGA, F.R.; SOARES, F.E.F.; GIUBERTI, T.Z. et al. Nematocidal activity of extracellular enzymes produced by the nematophagous fungus Duddingtonia flagrans on cyathostomin infective larvae.Veterinary Parasitology,v.212, n.3, p.214-218, 2015.

BUZATTI, A.; SANTOS, C.P.; FERNANDES, M.A.M. et al. Duddingtonia flagrans in the control of gastrointestinal nematodes of horses. Experimental Parasitology, v.159, p.14, 2015.

CANEVER, R.J.; BRAGA, P.R.; $\mathrm{BOECKH}, \mathrm{A}$. et al. Lack of Cyathostomin 
sp. reduction after anthelmintic treatment in horses in Brazil.Veterinary Parasitology, v.194, n.1, p.35-39, 2013. CAMPOS, A.K.; ARAÚJO, J.V.; GUIMARÃES, M.P. Interaction between the nematophagous fungus Duddingtonia flagrans and infective larvae of Haemonchus contortus (Nematoda: Trichostrongyloidea). Journal of Helminthology, v.82, p.337-341, 2008.

ABRAHÃO, C. L. H. (2016). Desenvolvimento corporal e sua relação com o número de ovos de ciatostomíneos nas fezes em equinos jovens da raça puro sangue inglês. 2016. Curitiba, 70f. Dissertação (Mestrado em Ciências Veterinárias) Curso de Pós-graduação em Ciências Veterinárias, Universidade Federal do Paraná, Curitiba.

CEZAR, A.S.; CATTO, J.B.; BIANCHIN, I. Alternative control of the gastrointestinal nematodes of the ruminants: actuality and perspectives. Ciência Rural, v.38, n.7, p.2083-2091, 2008.

COBB, R.; BOECKH, A. Moxidectin: a review of chemistry, pharmacokinetics and use in horses. Parasites \& Vectors, v.2, Supl.2, S5, 2009.

CORNING, S. Equine cyathostomins: a review of biology, clinical significance and therapy. Parasites \& Vectors, v.2, Supl.2, S1 2009.

COURTNEY, C.H.; ASQUITH, R.L. Seasonal changes in pasture infectivity by equine cyathostomes in north central Florida. Equine Veterinary Journal, v.17, n.3, p.240-242, 1985.

CRUZ, D.G.; ARAÚJO, F.B.; MOLENTO, M.B. et al. Kinetics of capture and infection of infective larvae of trichostrongylides and free-living nematodes Panagrellus sp. by Duddingtonia flagrans. Parasitology Research, v.109, n.4, p.1085-1091, 2011.
CRUZ, D.G.; COSTA, L.M.; ROCHA, L.O. ET AL. Serine proteases activity is important for the interaction of nematophagous fungus Duddingtonia flagrans with infective larvae of trichostrongylides and free-living nematodes Panagrellus spp. Fungal Biology, v.119, n.8, p.672-678, 2015.

DIAS, A.S.; ARAÚJO, J.V.; CAMPOS, A.K. et al. Application of a formulation of the nematophagous fungus Duddingtonia flagrans in the control of cattle gastrointestinal nematodiosis. World Journal of Microbiology and Biotechnology, v.23, n.9, p.1245-1252, 2007.

FAEDO, M.; LARSEN, M.; WALLER, P.J. The potential of nematophagous fungi to control the free-living stages of nematode parasites of sheep: Comparison between Australian isolates of Arthrobotrys spp. and Duddingtonia flagrans. Veterinary Parasitology, v.72, n.2, p.149-155, 1997.

FAEDO, M.; BARNES, E.H.; DOBSON, R.J. et al. The potential of nematophagous fungi to control the freeliving stages of nematode parasites of sheep: Pasture plot study with Duddingtonia flagrans. Veterinary Parasitology, v.76, n.1, p.129-135, 1998.

FERNÁNDEZ, A.S.; LARSEN, M.; NANSEN, $P$. et al. Effect of the nematode-trapping fungus Duddingtonia flagrans on the free-living stages of horse parasitic nematodes: a plot study.Veterinary Parasitology, v.73, n.3, p.257-266, 1997.

FERNÁNDEZ, A.S.; HENNINGSEN, E.; LARSEN, M. et al. A new isolate of the nematophagous fungus Duddingtonia flagrans a biological control agent against free-living larvae of horse strongyles. Equine Veterinary Journal, v.31, p. 488-491, 1999.

GAWOR, J.J. The prevalence and abundance of internal parasites in 
working horses autopsied in Poland. Veterinary Parasitology, v.58, p.99108. 1995.

GEURDEN, T.; VAN DOORN, D.; CLAEREBOUT, E. et al. Decreased strongyle egg re-appearance period after treatment with ivermectin and moxidectin in horses in Belgium, Italy and The Netherlands. Veterinary Parasitology, v.204, n.3, p.291-296, 2014.

GRONVOLD, J.; WOLSTRUP, J.; NANSEN, P. et al. Biological control of nematode parasites in cattle with nematode-trapping fungi: a survey of Danish studies. Veterinary Parasitology, v.48, n.1, p.311-325, 1993.

GRONVOLD, J.; NANSEN, P.; HENRIKSEN, S.A. et al. Induction of traps by Ostertagia ostertagi larvae, chlamydospore production and growth rate in the nematode-trapping fungus Duddingtonia flagrans. Journal of Helminthology, v.70, p.291-297, 1996.

HERD, R.P.; WILLARDSON, K.L.; GABEL, A.A. Epidemiological approach to the control of horse strongyles. Equine Veterinary Journal, v.17, n.3, p.202207, 1985.

HERD, R.P. Equine parasite control solutions to anthelmintic associated problems. Equine Veterinary Education, v.2, n.2, p.86-91, 1990.

HERNÁNDEZ, J.Á.; ARROYO, F.L.; SUÁREZ, J. et al. Feeding horses with industrially manufactured pellets with fungal spores to promote nematode integrated control.

Parasitology, v.229, p.37-44, 2016.

JACOBS, D.; FOX, M.; GIBBONS, L. et al. In Principles of Veterinary Parasitology. Oxford: Willey Blackwell, 2016. 312p.

JANSSON, H.B.; NORDBRING-HERTZ, $B$. Interactions between nematophagous fungi and plant-parasitic nematodes: attraction, induction of trap formation and capture. Nematologica, v.26, n.4, p.383389, 1980.

KAPLAN, R.M. Anthelmintic resistance in nematodes of horses. Veterinary Research, v.33, p.491-507, 2002.

KAPLAN, R.M. Drug resistance in nematodes of veterinary importance: a status report. Trends Parasitology, v.20, p.477-481, 2004.

KAPLAN, R.M; NIELSEN, M.K. An evidence based approach to equine parasite control: It ain't the 60s anymore. Equine Veterinary Education, v.22, n.6, p.306-316, 2010.

KNOX, M.R.; JOSH, P.F.; ANDERSON, L.J. Deployment of Duddingtonia flagrans in an improved pasture system: dispersal, persistence, and effects on free-living soil nematodes and microarthropods. Biological Control, v.24, n.2, p.176-182, 2002.

KUZMINA, T.A.; KUZMIN, Y.I.; KHARCHENKO, V.A. Field study on the survival, migration and overwintering of infective larvae of horse strongyles on pasture in central Ukraine,Veterinary Parasitology, v.141, n.3-4, p.264-272, 2006.

LARSEN, M.; NANSEN, P.; WOLSTRUP, $\mathrm{J}$. et al. A. Biological control of trichostrongyles in calves by the fungus Duddingtonia flagrans fed to animals under natural grazing conditions. Veterinary Parasitology, v.60, n.3, p. 321-330, 1995.

LARSEN, M.; FAEDO, M.; WALLER, P.J. et al. The potential of nematophagous fungi to control the free-living stages of nematode parasites of sheep: Studies with Duddingtonia flagrans. Veterinary Parasitology, v.76, n.1, p.121-128, 1998.

LARSEN, M. Prospects for controlling animal parasitic nematodes by predacious micro fungi. Parasitology, v.120, n.7, p.121-131, 2000. 
LARSEN, M. Biological control of nematode parasites in sheep. Journal Animal Science, v.84, n.13, p.133-139, 2006.

LICHTENFELS, J.R.; KHARCHENKO, V.A.; KRECEK, R.C. et al. An annotated checklist by genus and species, of 93 species level names for 51 recognized species of small strongyles (Nematoda: Strongyloidea: Cyathostominea) of horses, asses and zebras of the world. Veterinary Parasitology, v.79, p.65-79, 1998.

LICHTENFELS, J.R.; KHARCHENKO, V.A.; DVOJNOS, G.M. Illustrated identification keys to strongylid parasites (Strongylidae: Nematoda) of horses, zebras and asses (Equidae).Veterinary parasitology, v.156, n.1, p.4-161, 2008.

LLOYD, S. Effects of previous control programmes on the proportion of horses shedding small numbers of strongyletype eggs. Veterinary Record: Journal of the British Veterinary Association, v.164, n.4, 2009.

LYSEK, H.; STERBA, J. Colonization of Ascaris lumbricoides eggs by the fungus Verticillium chlamydosporium Goddard. Folia Parasitologica, v.38, p.255-259, 1991.

LOVE, S.; MURPHY, D.; MELLOR, D. Pathogenicity of cyathostome infection.Veterinary Parasitology, v.31, n.85, p.113-122, 1999.

LOON, G.V.; DEPREZ, P.; MUYLLE, E. et al. Larval cyathostomiasis as a cause of death in two regularly dewormed horses. Journal of Veterinary Medicine Series A, v. 42, n. 110, p.301-306, 1995.

MADEIRA DE CARVALHO, L.M. Estrongilidoses dos equídeos - aspectos da sua epidemiologia, terapêutica e controlo. Revista semestral da Associação dos Estudantes da Faculdade de Medicina Veterinária, n.58, p.6-15, 2003.
MADEIRA DE CARVALHO, L.M.; GILLESPIE, A.T.; SERRA, P.M. et al. Eficácia do fungo nematófago Duddingtonia flagrans no controlo biológico da estrongilidose equina no Ribatejo. Revista Portuguesa de Ciências Veterinárias, v.102, p.233247, 2007.

MADEIRA DE CARVALHO, L.M.; SERRA, P.M.; BERNARDO, F.A. et al. Controlo integrado da estrongilidose equina com anti-helmínticos associados ao fungo Duddingtonia flagrans. Aspectos da sua utilização em Portugal. Acta Parasitológica Portuguesa,v.18, n.1/2, p.63-90, 2011.

MEYER, W.J.; WIEBE, M.G. Enzyme production by the nematode-trapping fungus, Duddingtonia flagrans. Biotechnology Letters, v.25, n.10, p.791-795, 2003.

MOLENTO, M.B. Resistência parasitária em helmintos de equídeos e propostas de manejo. Ciência Rural, v.35, n.6, p.1469-1477, 2005.

MOLENTO, M.B. Parasite control in the age of drug resistance and changing agricultural practices. Veterinary

Parasitology, v.163, n.3, p.229-234, 2009.

MOLENTO, M.B.; BUZATTI, A.; SPRENGER, L.K. Pasture larval count as a supporting method for parasite epidemiology, population dynamic and control in ruminants. Livestock Science, v.192, p.48-54, 2016.

MOTA, M.D.A.; CAMPOS, A.K.; ARAÚJO, J.V.D. Controle biológico de helmintos parasitos de animais: estágio atual e perspectivas futuras. Pesquisa Veterinária Brasileira, v.23, n.3, p.93100, 2003.

NIELSEN, M.K.; MONRAD, J.; OLSEN, S.N. Prescription-only anthelmintics-a questionnaire survey of strategies for surveillance and control of equine 
strongyles in Denmark. Veterinary Parasitology, v.135, n.1, p.47-55, 2006.

NIELSEN, M. K. Restrictions of anthelmintic usage: perspectives and potential consequences. Parasites \& Vectors, v.2, n.2, 2009.

NIELSEN, M.K. Sustainable equine parasite control: perspectives and research needs. Veterinary Parasitology, v.185, n.1, p.32-44, 2012.

NIELSEN, M.K.; PFISTER, K.; VON SAMSON-HIMMELSTJERNA,

Selective therapy in equine parasite control - Application and limitations.Veterinary

Parasitology, v.202, n.3, p.95-103, 2014.

NORDBRING-HERTZ

B.I.

Peptide-Induced Morphogenesis in the Nematode-Trapping Fungus Arthrobotrys oligospora. Physiologia Plantarum, v. 29, n.2, p.223-33, 1973.

OJEDA-ROBERTOS, N.F.; TORRESACOSTA, J. F.; AGUILAR-CABALLERO, A.J. et al. Assessing the efficacy of Duddingtonia flagrans chlamydospores per gram of faeces to control Haemonchus contortus larvae. Veterinary Parasitology, v.158, n.4, p. 329-335, 2008.

PAZ-SILVA, A.; FRANCISCO, I.; VALERO-COSS, R.O. et al. Ability of the fungus Duddingtonia flagrans to adapt to the cyathostomin egg-output by spreading chlamydospores. Veterinary Parasitology, v.179, p.277-282, 2011.

PEREGRINE, A.S.; MOLENTO, M.B.; KAPLAN, R.M. et al. Anthelmintic resistance in important parasites of horses: does it really matter? Veterinary

Parasitology, v.201, n.1, p.1-8, 2014.

PRAMER, D. Nematode trapping fungi. Science, v.144, p.382-388, 1964.

PROUDMAN, C.; MATTHEWS, J. Control of intestinal parasites in horses. In Practice, v. 22, n., p.90-97, 2000.

REINEMEYER, C.R. Controlling strongyle parasites of horses: a mandate for change. Proceedings of the 55th annual convention the American Association of Equine Practitioners, p.353-360, 2009.

REINEMEYER, C.R.; PRADO, J.C.; ANDERSEN, U.V. et al. Effects of daily pyrantel tartrate on strongylid population dynamics and performance parameters of young horses repeatedly infected with cyathostomins and Strongylus vulgaris. Veterinary

Parasitology, v.204, n.3, p.229-237, 2014.

SANGSTER, N.; BATTERHAM, P.; CHAPMAN, H.D. et al. Resistance to antiparasitic drugs: the role of molecular diagnosis. International Journal for Parasitology, v.32, p. 637-653, 2002.

SANTOS, C.P.; PADILHA, T.; RODRIGUES, M.L.A. Predatory activity of Arthrobotrys oligospora and Duddingtonia flagrans on pre-parasitic larval stages of cyathostominae under different constant temperatures. Ciência Rural, v.31, n.5, p.839-842, 2001.

SILVA, M.E.D.; ARAÚJO, J.V.D.; BRAGA, F.R. et al. Control of infective larvae of gastrointestinal nematodes in heifers using different isolates of nematophagous fungi. Revista Brasileira de Parasitologia Veterinária, v.22, n.1, p.78-83, 2013.

SOUZA, L.S.; SANTOS, C.N.; QUINELATO, S. et al. Development of cyathostomin eggs (NematodaCyathostominae) in different temperatures. Ciência Animal, v.22, n.2, p.17-24, 2012.

STIRLING, G.R.; WEST, L.M. Fungal parasites of root-knot nematodes eggs from tropical and sub-tropical regions of Australia. Australasian

Plant

Pathology, v.20, n.4, p.149-154, 1991. 
TAVELA, A.E.O.; ARAÚJO, J.V.; BRAGA, F.R. et al. Coadministration of sodium alginate pellets containing the fungi Duddingtonia flagrans and Monacrosporium thaumasium on cyathostomin infective larvae after passing through the gastrointestinal tract of horses. Research Veterinary Science, v.94, n.3, p.568-72, 2013.

TAYLOR, M.A.; COOP, R.L.; WALL, R.L. Parasitologia Veterinária. 3ed. Rio de Janeiro: Editora Guanabara Koogan, 2007. 768p.

TRAVERSA, D. The Little known Scenario of Anthelmintic Resistance in Equine Cyathostomes in Italy. Annals of the New York Academy of Sciences, v.1149, n.1, p.167-169, 2008.

UHLINGER, C. Uses of fecal egg count data in equine practice. Compendium on Continuing Education for the Practising Veterinary, v.15, p.742-749, 1993.

WAGHORN, T.S.; LEATHWICK, D.M.; CHEN, L.Y. et al. Efficacy of the nematode-trapping fungus Duddingtonia flagrans against three species of gastrointestinal nematodes in laboratory faecal cultures from sheep and goats. Veterinary Parasitology, v.118, n.3, p.227-234, 2003.

WALLER, P.J.; FAEDO, M. The prospects for biological control of the free-living stages of nematode parasites of livestock. International Journal for Parasitology, v.26, n.8, p.915-925, 1996.

WANG, R.B.; YANG, J.K.; LIN, C. et al. Purification and characterization of an extracellular serine protease from the nematode trapping fungus Dactylella shizishanna. Letters in Applied Microbiology, v.42, n.6, p.589-594, 2006.

WHARTON, D.A.; MURRAY, D.S. Carbohydrate/lectin interactions between the nematophagous fungus Arthrobotrys oligospora and the infective juveniles of Trichostrongylus colubriformis (Nematoda). Parasitology, v.101, n.1, p.101-106, 1990.

WOOTTON, L.M.O.; PRAMER, D. Valine-induced morphogenesis in Arthrobotrys conoides. Bacteriological Proceedings, v. 75, p. 225-232. 1966. 